\title{
Correlation for the solubilities of pharmaceutical compounds in supercritical carbon dioxide
}

\author{
Chie-Shaan Su, Yan-Ping Chen* \\ Department of Chemical Engineering, National Taiwan University, Taipei, Taiwan, ROC \\ Received 23 December 2006; received in revised form 28 February 2007; accepted 1 March 2007 \\ Available online 6 March 2007
}

\begin{abstract}
The solid solubilities of pharmaceutical compounds in supercritical carbon dioxide were correlated using the regular solution model with the Flory-Huggins equation. The pharmaceutical compounds include steroids, antioxidants, antibiotics, analgesics and specific functional drugs. The molar volumes of these solid solutes in supercritical carbon dioxide were taken as the empirical parameters in this study. They were optimally fitted for each pharmaceutical compound using the experimental solid solubility data from literature. The logarithms of the molar volumes of these solutes were then correlated as a linear function of the logarithms of the densities for supercritical carbon dioxide. With one or two parameters in this linear equation, satisfactory solid solubilities were calculated that were comparable to those from the commonly used semi-empirical equations with more adjustable parameters. The parameters of this model were further generalized as a function of the properties of the pharmaceutical compounds. It was observed that the prediction of solubilities of pharmaceutical compounds in supercritical carbon dioxide was within acceptable accuracy for more than $50 \%$ of the systems investigated in this study.
\end{abstract}

(C) 2007 Elsevier B.V. All rights reserved.

Keywords: Correlation; Solid solubility; Pharmaceutical compounds; Supercritical carbon dioxide

\section{Introduction}

Many applications of supercritical fluid technology have continuously been developed for the processing of food, pharmaceutical, polymer and specific chemicals [1,2]. Supercritical fluid technology for pharmaceutical compounds includes the modifications of particle size, shape, morphology and surface structure. It maximizes the drug efficiency and leads to a better option than conventional manufacturing processes. Carbon dioxide is the most commonly used supercritical fluid owing to its mild critical properties, nontoxic and inflammatory characteristics. There are several methods of pharmaceutical particle formation using supercritical $\mathrm{CO}_{2}$, such as RESS, SAS, SEDS and PGSS [3]. The major criterion for choosing different processes depends on the solubility of pharmaceutical compound in supercritical $\mathrm{CO}_{2}$. Experimental measurements of the solubilities of these substances in supercritical $\mathrm{CO}_{2}$ provided essential information for engineering processing. Increasing data are

\footnotetext{
* Corresponding author. Fax: +8862 23623040 .

E-mail address: ypchen@ ntu.edu.tw (Y.-P. Chen).
}

appearing in recent literature and it was the purpose of this study to develop a useful correlation based on these data of pharmaceutical compounds.

Calculations for the solubilities of pharmaceutical solid compounds were presented using equation of state, solution model, and semi-empirical equation methods. The equation of state approach was limited by the uncertain critical properties and sublimation pressures of complex pharmaceutical molecules. Semi-empirical equations were mostly often employed in literature. For example, Chrastil [4] derived an equation that was based on molecular association. Mendez-Santiago and Teja [5] developed a relationship for solid solubility that incorporated the Clausius-Clapeyron equation. Zhong et al. [6] proposed a model that the solute-solvent clusters were in chemical equilibrium with the free solute and solvent molecules. These correlation equations contained three or four constants that were empirically adjusted for each pure compound. No generalization of these parameters with the solute properties was attempted.

An alternative and feasible method for correlating the solubilities of complex pharmaceutical compounds in supercritical $\mathrm{CO}_{2}$ was the application of solution model. In this approach, the supercritical $\mathrm{CO}_{2}$ was taken as the liquid solvent, and an 
infinite dilution activity coefficient was employed to account for the nonideal behavior of solid-liquid equilibrium. Iwai et al. [7] correlated the high boiling point components in supercritical $\mathrm{CO}_{2}$ using the regular solution model coupled with the Flory-Huggins term. Bush and Eckert [8] presented a predictive model based on linear solvation energy relationship (LSER), but relatively large error around $100 \%$ existed in their model for polar compounds. In our previous study [9], solid solubility of biological compounds including steroids, antioxidants and xanthines in supercritical carbon dioxide were correlated as an extension of the model of Iwai et al. [7]. In recent years, more experimental data have been published in literature for the solubilities of pharmaceutical compounds in supercritical $\mathrm{CO}_{2}$. It was our intention to revise our previous correlation and investigate a better generalization of the model parameters. This correlation involved new experimental data of complex pharmaceutical components, and provided useful information for engineering applications. The feasibility for the prediction of the solubilities of pharmaceutical compounds in supercritical $\mathrm{CO}_{2}$ was also investigated.

\section{Method of calculation}

Appling the solution model, supercritical $\mathrm{CO}_{2}$ was treated as an expanded liquid. The equilibrium solubility of a solid solute (component 2), $y_{2}$, in supercritical $\mathrm{CO}_{2}$ (component 1) was expressed as:

$y_{2}=\frac{f_{2}^{\mathrm{s}}}{\gamma_{2}^{\infty} f_{2}^{1}}$

where $\gamma_{2}^{\infty}$ was the activity coefficient of the solid solute at infinite dilution, $f_{2}^{\mathrm{s}}$ and $f_{2}^{1}$ were the fugacities of pure solute in solid phase and supercritical phase, respectively. The ratio of these two fugacities was approximated as:

$\ln \frac{f_{2}^{\mathrm{s}}}{f_{2}^{1}}=\frac{\Delta H_{2}^{\mathrm{f}}}{R}\left(\frac{1}{T_{2, \mathrm{~m}}}-\frac{1}{T}\right)$

where $\Delta H_{2}^{\mathrm{f}}$ was the molar heat of fusion of the pharmaceutical compound, $T_{2, \mathrm{~m}}$ was its melting temperature, and $R$ was the gas constant. The infinite dilution activity coefficient $\gamma_{2}^{\infty}$ was expressed by the modified regular solution model coupled with the Flory-Huggins term:

$\ln \gamma_{2}^{\infty}=\left(\frac{v_{2}}{R T}\right)\left(\delta_{1}-\delta_{2}\right)^{2}+1-\left(\frac{v_{2}}{v_{1}}\right)+\ln \left(\frac{v_{2}}{v_{1}}\right)$

where $\delta$ was the solubility parameter, and $v$ was the molar volume:

$\delta_{i}=\left(\frac{\Delta U_{i}^{\mathrm{vap}}}{v_{i}}\right)^{0.5}$

where $\Delta U^{\text {vap }}$ in Eq. (4) was the molar internal energy of vaporization. Incorporating this infinite dilution activity coefficient and the fugacity ratio, the solubility of solid solute in supercrit- ical phase was:

$$
\begin{aligned}
\ln y_{2}= & \frac{\Delta H_{2}^{\mathrm{f}}}{R}\left(\frac{1}{T_{2, \mathrm{~m}}}-\frac{1}{T}\right)-\left(\frac{v_{2}}{R T}\right)\left(\delta_{1}-\delta_{2}\right)^{2}-1 \\
& +\left(\frac{v_{2}}{v_{1}}\right)-\ln \left(\frac{v_{2}}{v_{1}}\right)
\end{aligned}
$$

The heat of fusion, $\Delta H_{2}^{\mathrm{f}}$, in Eq. (5) was either taken from literature or estimated by the method of Yalkowsky [10]. The value of $\delta_{1}$ was directly calculated using the Peng-Robinson equation of state [11]. The molar volume of supercritical carbon dioxide, $v_{1}$, was estimated by Jacobsen and Stewart EOS with 32 constants regressed by Ely et al. [12]. $\delta_{2}$ was determined using $v_{2}$ and $\Delta U_{2}^{\mathrm{vap}}$, and the latter was estimated by the group contribution method developed by Fedor [13]. $v_{2}$ was taken as the adjustable parameter and was regressed for each solid solute by minimizing the objective function over all data points $j$ :

obj. $=\sum \frac{\left|y_{2, j}^{\mathrm{cal}}-y_{2, j}^{\exp }\right|}{y_{2, j}^{\exp }}$

The superscripts cal and exp denoted the calculated and experimental results, respectively.

To compare the calculated solubilities of pharmaceutical compounds from the solution model with those from semiempirical correlation, the following equations presented in literature were employed. The Chrastil equation [4] was:

$\ln c=k \ln \rho_{1}+\frac{a}{T}+b$

where $c$ was the concentration of solute in supercritical fluid with the unit of $\left(\mathrm{kg} / \mathrm{m}^{3}\right), \rho_{1}$ the density $\left(\mathrm{kg} / \mathrm{m}^{3}\right)$ of supercritical $\mathrm{CO}_{2}, k, a$, and $b$ were three adjustable parameters. The MendezSantiago and Teja equation [5] was:

$T \ln \left(y_{2} P\right)=a+b \rho_{1}+c T$

where $a, b$ and $c$ were also adjustable parameters. Finally, a simple two-parameter ( $a$ and $b$ ) equation cited in previous literature [14-16] was applied:

$\ln y_{2}=a \ln \rho_{1}+b$

The adjustable parameters in these semi-empirical equations were also optimally fitted in this study by minimizing the objective function of Eq. (6):

\section{Results and discussion}

Solid solubilities in supercritical carbon dioxide for 60 pharmaceutical compounds containing steroids, antioxidants, antibiotics, analgesics and specific functional drugs were correlated in this study. Table 1 lists these pharmaceutical compounds and their thermodynamic properties. The data sources for these pharmaceutical components are shown in Table 2. In this study, the solid solubilities were correlated using Eq. (5) where the molar volume $v_{2}$ of the solid solute in supercritical $\mathrm{CO}_{2}$ was taken as an adjustable parameter. The optimally fitted values of 
Table 1

Data references and physical properties for pharmaceutical compounds in this study

\begin{tabular}{|c|c|c|c|c|c|c|c|c|c|}
\hline Compound & Formula & $M_{\mathrm{W}}(\mathrm{g} / \mathrm{mol})$ & $T$ range $(\mathrm{K})$ & $\begin{array}{l}P \text { range } \\
(\mathrm{MPa})\end{array}$ & $\begin{array}{l}\text { Data } \\
\text { points }\end{array}$ & $T_{\mathrm{m}}(\mathrm{K})$ & $\begin{array}{l}\Delta H^{\mathrm{f}} \\
(\mathrm{kJ} / \mathrm{mol})\end{array}$ & $\begin{array}{l}\Delta U^{\mathrm{vap}, 298.15 \mathrm{~K}} \\
(\mathrm{~kJ} / \mathrm{mol})\end{array}$ & $\begin{array}{l}\text { Data } \\
\text { references }\end{array}$ \\
\hline Amical-48 & $\mathrm{C}_{8} \mathrm{H}_{8} \mathrm{O}_{2} \mathrm{SI}_{2}$ & 422.02 & $318-338$ & $10-30$ & 18 & 453.15 & 25.60 & 97.38 & 12 \\
\hline 9,10-Anthraquinone & $\mathrm{C}_{14} \mathrm{H}_{8} \mathrm{O}_{2}$ & 208.21 & $308-318$ & $8-31$ & 17 & 559.15 & 31.59 & 99.62 & 31 \\
\hline Artemisinin & $\mathrm{C}_{15} \mathrm{H}_{22} \mathrm{O}_{5}$ & 282.33 & $310-338$ & $10-27$ & 36 & 429.65 & 24.27 & 88.89 & 1 \\
\hline Ascorbyl palmitate & $\mathrm{C}_{22} \mathrm{H}_{38} \mathrm{O}_{7}$ & 414.53 & $308-313$ & $13-20$ & 8 & 389.65 & 79.09 & 206.42 & 25 \\
\hline Aspirin & $\mathrm{C}_{9} \mathrm{H}_{8} \mathrm{O}_{4}$ & 180.16 & $308-328$ & $12-25$ & 24 & 407.36 & 23.01 & 82.24 & 9 \\
\hline Beclomethasone dipropionate & $\mathrm{C}_{28} \mathrm{H}_{37} \mathrm{ClO}_{7}$ & 521.04 & $338-358$ & $21-39$ & 21 & 391.15 & 26.19 & 211.15 & 21 \\
\hline Benzocaine & $\mathrm{C}_{9} \mathrm{H}_{11} \mathrm{NO}_{2}$ & 165.19 & $308-348$ & $12-36$ & 40 & 363.05 & 20.51 & 67.80 & 8 \\
\hline Bisacodyl & $\mathrm{C}_{22} \mathrm{H}_{19} \mathrm{NO}_{4}$ & 361.39 & $308-348$ & $12-36$ & 39 & 408.27 & 23.06 & 152.00 & 3 \\
\hline (Rac) Boc-piperazine & $\mathrm{C}_{14} \mathrm{H}_{27} \mathrm{~N}_{3} \mathrm{O}_{3}$ & 285.38 & $308-328$ & $9-20$ & 19 & 381.05 & 21.52 & 106.73 & 5 \\
\hline (S) Boc-piperazine & $\mathrm{C}_{14} \mathrm{H}_{27} \mathrm{~N}_{3} \mathrm{O}_{3}$ & 285.38 & $308-328$ & $9-20$ & 21 & 376.33 & 21.26 & 106.73 & 5 \\
\hline Budesonide & $\mathrm{C}_{25} \mathrm{H}_{34} \mathrm{O}_{6}$ & 430.53 & $338-358$ & $21-39$ & 21 & 499.65 & 28.22 & 197.13 & 21 \\
\hline Caffeine & $\mathrm{C}_{8} \mathrm{H}_{10} \mathrm{~N}_{4} \mathrm{O}_{2}$ & 194.19 & $313-368$ & $8-35$ & 56 & 510.28 & 22.52 & 88.14 & $16,34,35$ \\
\hline Carbamazepine & $\mathrm{C}_{15} \mathrm{H}_{12} \mathrm{~N}_{2} \mathrm{O}$ & 236.27 & $308-348$ & $12-36$ & 39 & 464.22 & 26.22 & 107.61 & 20 \\
\hline Chlorothalonil & $\mathrm{C}_{8} \mathrm{Cl}_{4} \mathrm{~N}_{2}$ & 265.91 & $318-338$ & $10-30$ & 23 & 523.40 & 29.56 & 129.16 & 12 \\
\hline Cholesterol & $\mathrm{C}_{27} \mathrm{H}_{46} \mathrm{O}$ & 386.65 & $313-333$ & $10-25$ & 24 & 421.16 & 28.19 & 147.38 & 23,26 \\
\hline Cholesteryl acetate & $\mathrm{C}_{29} \mathrm{H}_{48} \mathrm{O}_{2}$ & 428.69 & $308-328$ & $9-24$ & 24 & 387.94 & 25.97 & 140.29 & 23 \\
\hline Cholesteryl benzoate & $\mathrm{C}_{34} \mathrm{H}_{50} \mathrm{O}_{2}$ & 490.76 & $308-328$ & $12-27$ & 20 & 421.75 & 28.23 & 167.51 & 23 \\
\hline Cholesteryl butyrate & $\mathrm{C}_{31} \mathrm{H}_{52} \mathrm{O}_{2}$ & 456.76 & $308-328$ & $10-24$ & 20 & 372.15 & 24.91 & 150.16 & 23 \\
\hline Codeine & $\mathrm{C}_{18} \mathrm{H}_{21} \mathrm{NO}_{3}$ & 299.36 & $308-348$ & $12-36$ & 45 & 429.40 & 24.25 & 124.81 & 20 \\
\hline$p$-Coumaric acid & $\mathrm{C}_{9} \mathrm{H}_{8} \mathrm{O}_{3}$ & 164.16 & $313-333$ & $9-50$ & 29 & 486.48 & 27.48 & 97.95 & 19 \\
\hline DDT & $\mathrm{C}_{14} \mathrm{H}_{9} \mathrm{Cl}_{5}$ & 354.48 & $313-333$ & $10-21$ & 18 & 381.65 & 51.56 & 114.43 & 27 \\
\hline Diazepam & $\mathrm{C}_{16} \mathrm{H}_{13} \mathrm{ClN}_{2} \mathrm{O}$ & 284.74 & $308-348$ & $12-36$ & 45 & 401.90 & 22.70 & 123.66 & 20 \\
\hline 7,8-Dihydroxy flavone & $\mathrm{C}_{15} \mathrm{H}_{10} \mathrm{O}_{4}$ & 254.24 & $308-318$ & $9-25$ & 9 & 558.10 & 34.50 & 153.80 & 7 \\
\hline Eflucimibe & $\mathrm{C}_{29} \mathrm{H}_{43} \mathrm{NO}_{2} \mathrm{~S}$ & 469.73 & $308-318$ & $8-30$ & 20 & 403.15 & 60.72 & 209.97 & 14 \\
\hline Erythromycin & $\mathrm{C}_{37} \mathrm{H}_{67} \mathrm{NO}_{13}$ & 733.93 & $313-333$ & $10-30$ & 8 & 464.40 & 26.23 & 347.88 & 16 \\
\hline Ferulic acid & $\mathrm{C}_{10} \mathrm{H}_{10} \mathrm{O}_{4}$ & 194.18 & $313-333$ & $12-28$ & 18 & 445.15 & 25.14 & 106.00 & 4 \\
\hline Flurbiprofen & $\mathrm{C}_{15} \mathrm{H}_{13} \mathrm{FO}_{2}$ & 244.26 & $303-323$ & $8-25$ & 27 & 383.90 & 21.68 & 105.29 & 2 \\
\hline Ketoprofen & $\mathrm{C}_{16} \mathrm{H}_{14} \mathrm{O}_{3}$ & 254.28 & $313-332$ & $9-25$ & 25 & 367.15 & 20.74 & 116.96 & 17,30 \\
\hline Medroxyprogesterone acetate & $\mathrm{C}_{24} \mathrm{H}_{34} \mathrm{O}_{4}$ & 386.54 & $308-348$ & $11-36$ & 48 & 480.60 & 27.15 & 141.73 & 13 \\
\hline Methimazole & $\mathrm{C}_{4} \mathrm{H}_{6} \mathrm{~N}_{2} \mathrm{~S}$ & 114.17 & $308-348$ & $12-36$ & 40 & 418.82 & 23.66 & 49.02 & 15 \\
\hline Methyl gallate & $\mathrm{C}_{8} \mathrm{H}_{8} \mathrm{O}_{5}$ & 184.15 & $313-333$ & $10-50$ & 27 & 475.32 & 26.85 & 143.99 & 18 \\
\hline Methylparaben & $\mathrm{C}_{8} \mathrm{H}_{8} \mathrm{O}_{3}$ & 152.15 & $308-348$ & $12-36$ & 40 & 402.27 & 24.31 & 84.41 & 3 \\
\hline Metronidazole benzoate & $\mathrm{C}_{13} \mathrm{H}_{13} \mathrm{~N}_{3} \mathrm{O}_{4}$ & 275.27 & $308-348$ & $12-36$ & 40 & 375.15 & 21.19 & 123.66 & 8 \\
\hline 1,4-Naphthoquinone & $\mathrm{C}_{10} \mathrm{H}_{6} \mathrm{O}_{2}$ & 158.15 & $318-343$ & $10-36$ & 18 & 399.15 & 15.41 & 77.99 & 28 \\
\hline Naproxen & $\mathrm{C}_{14} \mathrm{H}_{14} \mathrm{O}_{3}$ & 230.26 & $308-348$ & $9-36$ & 58 & 427.24 & 31.50 & 97.36 & 8,24 \\
\hline Nifedipine & $\mathrm{C}_{17} \mathrm{H}_{18} \mathrm{~N}_{2} \mathrm{O}_{6}$ & 346.33 & $333-373$ & $13-30$ & 29 & 446.00 & 25.20 & 215.52 & 29 \\
\hline Nimesulide & $\mathrm{C}_{13} \mathrm{H}_{12} \mathrm{~N}_{2} \mathrm{O}_{5} \mathrm{~S}$ & 308.31 & $313-332$ & $13-22$ & 8 & 421.65 & 23.82 & 116.46 & 30 \\
\hline Nimodipine & $\mathrm{C}_{21} \mathrm{H}_{26} \mathrm{~N}_{2} \mathrm{O}_{7}$ & 418.44 & $313-333$ & $10-25$ & 21 & 398.15 & 26.65 & 153.53 & 6 \\
\hline Penicillin G & $\mathrm{C}_{16} \mathrm{H}_{18} \mathrm{~N}_{2} \mathrm{O}_{4} \mathrm{~S}$ & 334.39 & $313-333$ & $10-35$ & 18 & $736.31^{\mathrm{b}}$ & 41.59 & 151.25 & 22 \\
\hline 3,3,4,5,7-Pentahydroxy flavone & $\mathrm{C}_{15} \mathrm{H}_{10} \mathrm{O}_{7}$ & 302.24 & $308-318$ & $10-25$ & 8 & 587.15 & 40.90 & 243.17 & 7 \\
\hline Phenazopyridine & $\mathrm{C}_{11} \mathrm{H}_{11} \mathrm{~N}_{5}$ & 213.24 & $308-348$ & $12-36$ & 45 & 412.15 & 23.28 & 97.19 & 15 \\
\hline Piroxicam & $\mathrm{C}_{15} \mathrm{H}_{13} \mathrm{~N}_{3} \mathrm{O}_{4} \mathrm{~S}$ & 331.35 & $313-332$ & $10-22$ & 9 & 469.15 & 26.51 & 167.84 & 30 \\
\hline Progesterone & $\mathrm{C}_{21} \mathrm{H}_{30} \mathrm{O}_{2}$ & 314.46 & $313-333$ & $9-24$ & 11 & 400.15 & 26.89 & 119.47 & 32 \\
\hline Propranolol & $\mathrm{C}_{16} \mathrm{H}_{21} \mathrm{NO}_{2}$ & 259.35 & $308-348$ & $12-36$ & 45 & 369.15 & 28.57 & 121.21 & 15 \\
\hline Propyl gallate & $\mathrm{C}_{10} \mathrm{H}_{12} \mathrm{O}_{5}$ & 212.20 & $313-333$ & $15-25$ & 8 & 423.15 & 23.91 & 130.02 & 25 \\
\hline Protocatechualdehyde & $\mathrm{C}_{7} \mathrm{H}_{6} \mathrm{O}_{3}$ & 138.12 & $313-333$ & $10-50$ & 24 & 426.48 & 24.09 & 112.84 & 18 \\
\hline Protocatechuic acid & $\mathrm{C}_{7} \mathrm{H}_{6} \mathrm{O}_{4}$ & 154.12 & $313-333$ & $10-50$ & 24 & 472.98 & 26.72 & 119.12 & 18 \\
\hline$p$-Quinone & $\mathrm{C}_{6} \mathrm{H}_{4} \mathrm{O}_{2}$ & 108.09 & $308-318$ & $9-29$ & 18 & 390.15 & 22.04 & 58.03 & 31 \\
\hline Salicylic acid & $\mathrm{C}_{7} \mathrm{H}_{6} \mathrm{O}_{3}$ & 138.12 & $313-328$ & $9-25$ & 11 & 432.24 & 24.41 & 89.33 & 17 \\
\hline Stigmasterol & $\mathrm{C}_{29} \mathrm{H}_{48} \mathrm{O}$ & 412.69 & $308-333$ & $9-30$ & 19 & 435.15 & 29.14 & 155.94 & 33 \\
\hline Sulfadimethoxine & $\mathrm{C}_{12} \mathrm{H}_{14} \mathrm{~N}_{4} \mathrm{O}_{4} \mathrm{~S}$ & 310.33 & $313-333$ & $13-49$ & 19 & 475.90 & 26.88 & 139.54 & 10 \\
\hline Sulfamerazine & $\mathrm{C}_{11} \mathrm{H}_{12} \mathrm{~N}_{4} \mathrm{O}_{2} \mathrm{~S}$ & 264.30 & $313-333$ & $15-47$ & 18 & 509.75 & 31.60 & 128.14 & 10 \\
\hline Syringic acid & $\mathrm{C}_{9} \mathrm{H}_{10} \mathrm{O}_{5}$ & 198.17 & $313-333$ & $9-50$ & 27 & 478.90 & 27.05 & 105.44 & 11 \\
\hline Tebuconazole & $\mathrm{C}_{16} \mathrm{H}_{22} \mathrm{ClN}_{3} \mathrm{O}$ & 307.83 & $323-338$ & $10-30$ & 12 & 377.85 & 21.34 & 142.40 & 12 \\
\hline Theobromine & $\mathrm{C}_{7} \mathrm{H}_{8} \mathrm{~N}_{4} \mathrm{O}_{2}$ & 180.16 & $313-353$ & $19-35$ & 23 & 620.00 & 41.11 & 90.96 & 35 \\
\hline Theophylline & $\mathrm{C}_{7} \mathrm{H}_{8} \mathrm{~N}_{4} \mathrm{O}_{2}$ & 180.16 & $313-353$ & $20-35$ & 24 & 547.50 & 29.71 & 90.96 & 35 \\
\hline Uracil & $\mathrm{C}_{4} \mathrm{H}_{4} \mathrm{~N}_{2} \mathrm{O}_{2}$ & 122.09 & $313-333$ & $10-30$ & 12 & 609.65 & 34.44 & 61.13 & 16 \\
\hline Vanillic acid & $\mathrm{C}_{8} \mathrm{H}_{8} \mathrm{O}_{4}$ & 168.15 & $313-333$ & $9-50$ & 28 & 482.61 & 27.26 & 97.38 & 11 \\
\hline Vitamin C & $\mathrm{C}_{6} \mathrm{H}_{8} \mathrm{O}_{6}$ & 176.12 & 313 & $13-20$ & 4 & 465.15 & 26.28 & 128.49 & 25 \\
\hline Zopiclone & $\mathrm{C}_{17} \mathrm{H}_{17} \mathrm{ClN}_{6} \mathrm{O}_{3}$ & 388.81 & $313-333$ & $10-25$ & 21 & 451.15 & 25.48 & 175.50 & 6 \\
\hline
\end{tabular}

${ }^{a}$ Data reference are listed in Table 2.

b Melting temperature estimate from the group contribution method of Joback et al. [17]. 
Table 2

Data sources for solubility data

\begin{tabular}{|c|c|}
\hline Reference number & Source \\
\hline 1 & J. Chem. Eng. Data 48 (2003) 330-332 \\
\hline 2 & J. Chem. Eng. Data 49 (2004) 449-452 \\
\hline 3 & J. Chem. Eng. Data 48 (2003) 61-65 \\
\hline 4 & J. Chem. Eng. Data 46 (2001) 1255-1257 \\
\hline 5 & J. Chem. Eng. Data 49 (2004) 1560-1564 \\
\hline 6 & J. Chem. Eng. Data 46 (2001) 1211-1214 \\
\hline 7 & J. Chem. Eng. Data 48 (2003) 1040-1043 \\
\hline 8 & J. Chem. Eng. Data 49 (2004) 709-712 \\
\hline 9 & J. Chem. Eng. Data 49 (2004) 1323-1327 \\
\hline 10 & J. Chem. Eng. Data 44 (1999) 1222-1225 \\
\hline 11 & J. Chem. Eng. Data 49 (2004) 779-782 \\
\hline 12 & J. Chem. Eng. Data 48 (2003) 541-547 \\
\hline 13 & J. Supercrit. Fluids 30 (2004) 111-117 \\
\hline 14 & J. Supercrit. Fluids 31 (2004) 133-140 \\
\hline 15 & J. Pharm. Biomed. Anal. 32 (2003) 181-187 \\
\hline 16 & Fluid Phase Equilib. 220 (2004) 57-69 \\
\hline 17 & J. Chem. Eng. Data 45 (2000) 161-165 \\
\hline 18 & J. Supercrit. Fluids 23 (2002) 113-121 \\
\hline 19 & J. Supercrit. Fluids 27 (2003) 239-245 \\
\hline 20 & J. Chem. Eng. Data 46 (2001) 451-455 \\
\hline 21 & J. Supercrit. Fluids 33 (2005) 21-25 \\
\hline 22 & J. Supercrit. Fluids 15 (1999) 183-190 \\
\hline 23 & J. Supercrit. Fluids 30 (2004) 25-39 \\
\hline 24 & Ind. Eng. Chem. Res. 32 (1993) 1471-1481 \\
\hline 25 & J. Supercrit. Fluids 14 (1999) 139-144 \\
\hline 26 & Ind. Eng. Chem. Res. 30 (1991) 2476-2482 \\
\hline 27 & Ind. Eng. Chem. Res. 33 (1994) 2757-2763 \\
\hline 28 & J. Chem. Eng. Data 31 (1986) 204-212 \\
\hline 29 & J. Chem. Eng. Data 40 (1995) 216-220 \\
\hline 30 & J. Chem. Eng. Data 41 (1996) 1083-1086 \\
\hline 31 & J. Chem. Eng. Data 42 (1997) 463-466 \\
\hline 32 & Ind. Eng. Chem. Res. 35 (1996) 4718-4726 \\
\hline 33 & Biotechnol. Prog. 2 (1986) 29-39 \\
\hline 34 & Fluid Phase Equilib. 68 (1991) 263-280 \\
\hline 35 & Fluid Phase Equilib. 95 (1994) 215-226 \\
\hline
\end{tabular}

$\ln v_{2}$ were observed as a linear function of the logarithm of density for supercritical $\mathrm{CO}_{2}$. The similar trend was also presented by Iwai et al. [7] and our previous study [9]. Fig. 1 shows an example for the plot of the optimal $\ln v_{2}$ values of Diazepam, a drug for the symptomatic relief of tension and anxiety, against the logarithm of density of supercritical $\mathrm{CO}_{2}$. A linear relationship was demonstrated and the similar results were obtained for other pharmaceutical compounds in this study. A simple correlation was then proposed:

$\ln v_{2}=\alpha \ln \rho_{1}+\beta$

where $\alpha$ and $\beta$ were two temperature independent parameters for each pharmaceutical compound.

Table 3 presents the optimally fitted $\alpha$ and $\beta$ parameters in the two-parameter model shown in Eq. (10) for various pharmaceutical compounds. Applying Eq. (10) into Eq. (5), the calculated absolute relative deviation in solid solubility (ARDY) was $16.5 \%$. Over $70 \%$ of the systems investigated in this study had ARDY less than $20 \%$ that was within the possible experimental accuracy.

A further simplification for the model parameters was attempted by setting either $\alpha$ or $\beta$ as a constant. An average

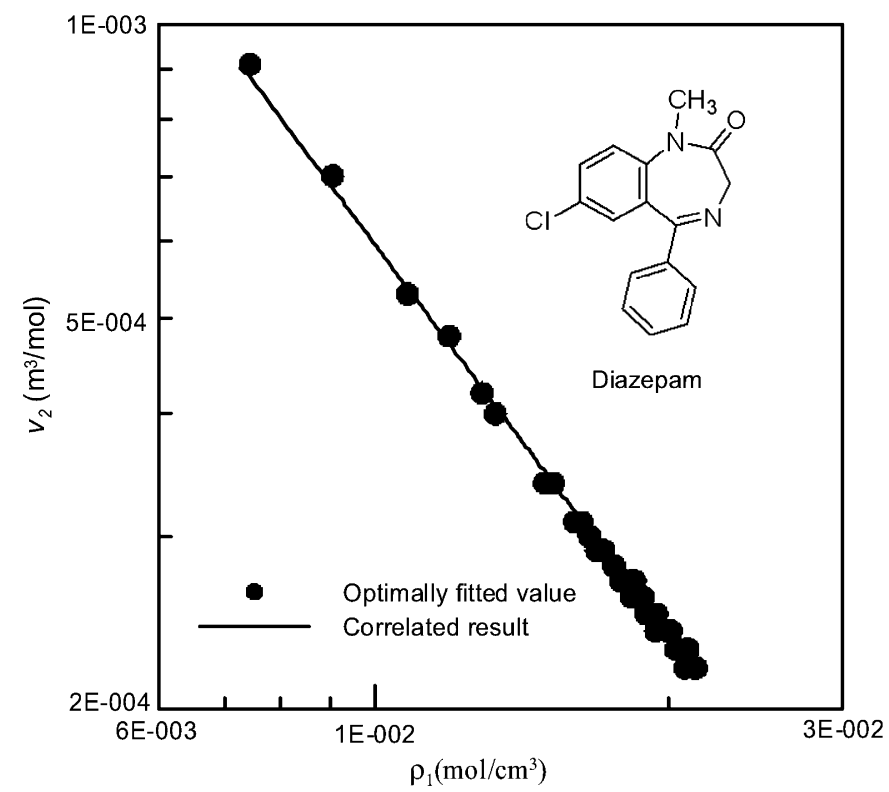

Fig. 1. Plot of the optimally fitted solid molar volume of diazepam (2) in supercritical $\mathrm{CO}_{2}$ (1) against density of pure $\mathrm{CO}_{2}$.

value of $\beta=-12.89$ for all compounds was applied in this study, and $\alpha$ was left as a single adjustable parameter. The results are shown in Table 3 as the one-parameter model with an ARDY of $23.7 \%$. With only one parameter, over $60 \%$ of the compounds had an ARDY less than $25 \%$ that was acceptable for the complex pharmaceutical molecules.

Fig. 2 shows the calculated solubility of Vanillic acid (an antioxidant) using either the two- or one-parameter solution model in this study. The ARDY were $12 \%$ and $20 \%$ from each model, respectively. The results in Fig. 2 showed that the solution model was feasible in correlating the experimental solubility data at various temperatures. Fig. 3 presents another example for a more complex molecule of nimodipine. The ARDY from the

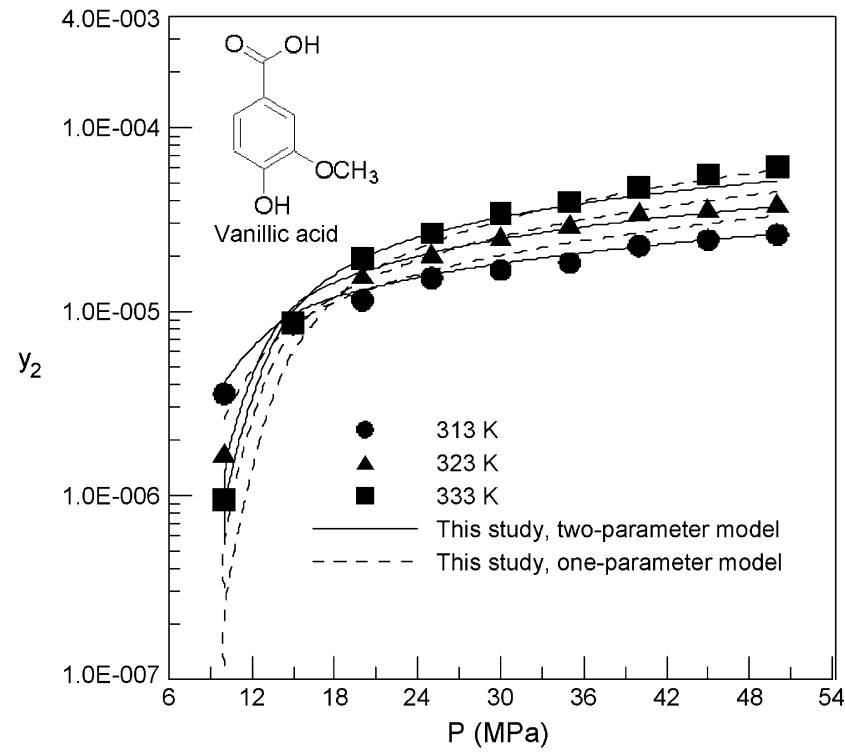

Fig. 2. Comparison of the experimental and calculated solid solubilities of vanillic acid (2) in supercritical $\mathrm{CO}_{2}(1)$ at various temperatures. 
Table 3

Calculation results for the solid solubility of pharmaceutical compounds in supercritical carbon dioxide using various models

\begin{tabular}{|c|c|c|c|c|c|c|c|c|}
\hline \multirow[t]{3}{*}{ Component } & \multicolumn{5}{|c|}{ This study } & \multicolumn{3}{|c|}{ Semi-empirical correlation equations } \\
\hline & \multicolumn{3}{|c|}{ Two-parameter model } & \multicolumn{2}{|c|}{ One-parameter model, $\beta=-12.89$} & \multirow{2}{*}{$\begin{array}{l}\text { I } \\
\text { ARDY (\%) }\end{array}$} & \multirow{2}{*}{$\begin{array}{l}\text { II } \\
\text { ARDY }(\%)\end{array}$} & \multirow{2}{*}{$\begin{array}{l}\text { III } \\
\text { ARDY (\%) }\end{array}$} \\
\hline & $\alpha$ & $\beta$ & $\operatorname{ARDY}(\%)$ & $\alpha$ & $\operatorname{ARDY}(\%)$ & & & \\
\hline Amical-48 & -1.31 & -13.72 & 8.1 & -1.10 & 28.0 & 8.8 & 10.0 & 32.0 \\
\hline 9,10-Anthraquinone & -0.96 & -12.16 & 16.9 & -1.14 & 39.2 & 12.8 & 12.3 & 34.1 \\
\hline Artemisinin & -1.20 & -13.16 & 8.1 & -1.13 & 9.2 & 6.2 & 6.3 & 23.1 \\
\hline Ascorbyl palmitate & -1.08 & -11.83 & 7.2 & -1.35 & 29.0 & 3.6 & 3.9 & 13.6 \\
\hline Aspirin & -1.24 & -13.71 & 11.5 & -1.04 & 19.7 & 5.2 & 4.7 & 30.3 \\
\hline Beclomethasone dipropionate & -1.25 & -12.61 & 11.0 & -1.32 & 13.6 & 10.8 & 10.7 & 24.8 \\
\hline Benzocaine & -1.21 & -13.61 & 18.9 & -1.03 & 32.3 & 10.7 & 11.7 & 39.1 \\
\hline Bisacodyl & -1.21 & -12.77 & 16.9 & -1.25 & 20.2 & 12.0 & 9.5 & 40.4 \\
\hline (Rac) Boc-piperazine & -1.30 & -13.51 & 17.5 & -1.14 & 30.2 & 11.6 & 10.5 & 30.6 \\
\hline (S) Boc-piperazine & -1.21 & -13.01 & 24.7 & -1.18 & 26.0 & 20.0 & 20.0 & 33.2 \\
\hline Budesonide & -1.22 & -12.50 & 11.5 & -1.31 & 17.3 & 11.5 & 11.2 & 26.7 \\
\hline Caffeine & -1.03 & -12.57 & 20.4 & -1.10 & 31.6 & 21.3 & 28.1 & 48.1 \\
\hline Carbamazepine & -1.15 & -13.04 & 18.4 & -1.11 & 18.8 & 13.4 & 13.8 & 43.6 \\
\hline Chlorothalonil & -1.20 & -12.78 & 22.6 & -1.23 & 23.7 & 19.8 & 19.6 & 28.1 \\
\hline Cholesterol & -1.21 & -12.76 & 7.0 & -1.24 & 8.6 & 6.0 & 6.2 & 24.6 \\
\hline Cholesteryl acetate & -1.18 & -12.67 & 15.4 & -1.24 & 24.9 & 10.1 & 9.3 & 30.8 \\
\hline Cholesteryl benzoate & -1.20 & -12.63 & 14.7 & -1.27 & 18.7 & 6.8 & 6.9 & 38.9 \\
\hline Cholesteryl butyrate & -1.16 & -12.48 & 7.3 & -1.25 & 28.5 & 6.4 & 7.3 & 29.4 \\
\hline Codeine & -1.23 & -13.01 & 14.2 & -1.20 & 16.6 & 12.6 & 11.3 & 42.3 \\
\hline$p$-Coumaric acid & -1.07 & -13.17 & 28.5 & -1.00 & 30.2 & 20.4 & 19.0 & 49.1 \\
\hline DDT & -1.12 & -12.57 & 13.3 & -1.20 & 26.5 & 2.8 & 7.6 & 25.4 \\
\hline Diazepam & -1.26 & -13.14 & 11.3 & -1.19 & 15.8 & 11.6 & 12.3 & 32.0 \\
\hline 7,8-Dihydroxy flavone & -1.41 & -13.42 & 13.5 & -1.28 & 26.8 & 4.5 & 8.5 & 29.1 \\
\hline Eflucimibe & -1.24 & -12.54 & 16.8 & -1.32 & 33.3 & 14.0 & 13.1 & 43.6 \\
\hline Erythromycin & -1.41 & -12.51 & 12.8 & -1.50 & 51.2 & 14.4 & 17.8 & 39.6 \\
\hline Ferulic acid & -1.22 & -13.43 & 6.1 & -1.08 & 15.2 & 5.4 & 6.6 & 41.9 \\
\hline Flurbiprofen & -1.17 & -13.14 & 21.0 & -1.11 & 23.8 & 8.4 & 9.8 & 38.6 \\
\hline Ketoprofen & -1.16 & -12.98 & 19.9 & -1.14 & 20.2 & 11.1 & 11.6 & 40.2 \\
\hline Medroxyprogesterone acetate & -1.16 & -12.57 & 17.2 & -1.24 & 22.7 & 17.5 & 16.8 & 37.5 \\
\hline Methimazole & -0.33 & -11.34 & 12.0 & -0.72 & 21.1 & 12.7 & 10.7 & 36.6 \\
\hline Methyl gallate & -1.25 & -13.14 & 13.3 & -1.18 & 17.2 & 10.8 & 8.7 & 45.1 \\
\hline Methylparaben & -1.27 & -13.73 & 11.5 & -1.06 & 26.2 & 9.5 & 9.3 & 39.1 \\
\hline Metronidazole benzoate & -1.21 & -12.92 & 16.7 & -1.20 & 16.9 & 17.2 & 14.6 & 37.2 \\
\hline 1,4-Naphthoquinone & -1.16 & -13.19 & 12.7 & -1.09 & 21.7 & 7.4 & 11.3 & 27.0 \\
\hline Naproxen & -1.17 & -13.22 & 14.5 & -1.09 & 20.2 & 14.6 & 14.9 & 45.5 \\
\hline Nifedipine & -1.22 & -13.00 & 15.7 & -1.19 & 18.5 & 14.2 & 16.8 & 35.9 \\
\hline Nimesulide & -1.12 & -12.75 & 20.0 & -1.15 & 22.4 & 8.0 & 8.3 & 38.4 \\
\hline Nimodipine & -1.31 & -13.21 & 11.5 & -1.23 & 25.7 & 7.4 & 9.2 & 31.9 \\
\hline Penicillin G & -1.20 & -12.45 & 31.2 & -1.31 & 36.7 & 24.9 & 25.0 & 48.7 \\
\hline 3,3,4,5,7-Pentahydroxy flavone & -1.37 & -12.71 & 8.0 & -1.41 & 23.5 & 4.4 & 5.1 & 25.7 \\
\hline Phenazopyridine & -1.12 & -13.09 & 15.8 & -1.08 & 17.5 & 10.7 & 8.9 & 43.2 \\
\hline Piroxicam & -1.20 & -12.58 & 8.1 & -1.28 & 24.9 & 8.3 & 8.1 & 33.4 \\
\hline Progesterone & -1.07 & -12.37 & 7.9 & -1.19 & 38.1 & 3.2 & 7.9 & 24.6 \\
\hline Propranolol & -1.28 & -13.32 & 25.5 & -1.17 & 35.0 & 17.9 & 16.9 & 52.3 \\
\hline Propyl gallate & -1.32 & -13.53 & 6.6 & -1.16 & 21.6 & 3.4 & 4.8 & 36.9 \\
\hline Protocatechualdehyde & -1.22 & -13.38 & 25.4 & -1.10 & 29.2 & 18.3 & 15.3 & 49.2 \\
\hline Protocatechuic acid & -1.16 & -13.19 & 27.0 & -1.09 & 30.7 & 15.6 & 12.8 & 46.6 \\
\hline$p$-Quinone & -1.12 & -13.15 & 21.6 & -1.05 & 23.0 & 15.3 & 17.3 & 31.7 \\
\hline Salicylic acid & -1.24 & -13.42 & 8.0 & -1.11 & 24.4 & 4.7 & 4.7 & 26.0 \\
\hline Stigmasterol & -1.24 & -12.87 & 20.7 & -1.24 & 20.9 & 13.2 & 12.3 & 46.7 \\
\hline Sulfadimethoxine & -0.92 & -11.92 & 39.0 & -1.17 & 46.6 & 28.5 & 27.8 & 29.5 \\
\hline Sulfamerazine & -0.89 & -11.82 & 29.8 & -1.16 & 50.3 & 28.6 & 29.5 & 41.9 \\
\hline Syringic acid & -1.03 & -12.71 & 14.8 & -1.08 & 17.3 & 8.9 & 19.4 & 39.6 \\
\hline Tebuconazole & -1.19 & -12.74 & 32.7 & -1.23 & 33.9 & 26.9 & 13.7 & 47.5 \\
\hline Theobromine & -1.03 & -12.65 & 15.9 & -1.09 & 16.4 & 10.3 & 11.7 & 29.6 \\
\hline Theophylline & -1.11 & -13.04 & 12.6 & -1.08 & 13.9 & 5.2 & 5.6 & 20.7 \\
\hline Uracil & -0.89 & -12.56 & 40.7 & -0.97 & 43.9 & 29.8 & 28.0 & 54.3 \\
\hline Vanillic acid & -1.21 & -13.40 & 12.0 & -1.09 & 20.0 & 10.2 & 11.4 & 33.3 \\
\hline Vitamin C & -1.22 & -12.99 & 2.0 & -1.20 & 3.7 & 2.0 & 1.4 & 2.0 \\
\hline Zopiclone & -1.37 & -13.24 & 5.4 & -1.28 & 22.1 & 6.6 & 7.6 & 30.1 \\
\hline Grand & & & 16.5 & & 23.7 & 12.7 & 13.0 & 37.3 \\
\hline
\end{tabular}

I: $\ln c=k \ln \rho_{1}+a / T+b$ (Chrastil equation [4]); II: $T \ln \left(y_{2} P\right)=a+b \rho_{1}+c T$ (Mendez-Santiago and Teja equation [5]); III: $\ln y_{2}=a \ln \rho_{1}+b$ (model employed in Refs. [14-16]). 


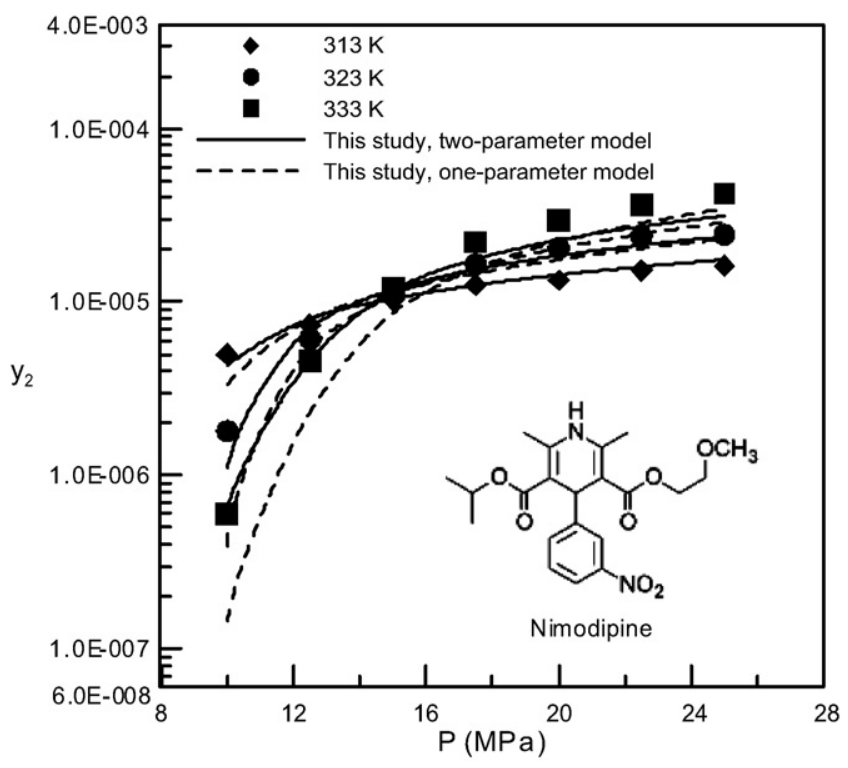

Fig. 3. Comparison of the experimental and calculated solid solubilities of nimodipine (2) in supercritical $\mathrm{CO}_{2}(1)$ at various temperatures.

two- or one-parameter model were $11.5 \%$ and $25.7 \%$, respectively. It is depicted that the single parameter model yielded relatively larger deviation than that from the dual parameter approach, but still gave the acceptable saturated solid solubility at higher-pressure range.

The calculation results from this study were compared with those from the semi-empirical equations, as shown in Table 3. Empirical model I was the Chrastil equation [4] with three adjustable parameters. The ARDY from the Chrastil equation was $12.7 \%$ that was comparable to the solution model with two parameters in this study. Empirical model II was the MendezSantiago and Teja equation [5], also with three adjustable parameters. It resulted in the similar accuracy (an ARDY of 13\%) as that from the Chrastil equation. Empirical model III [14-16] was a two-parameter equation that gave an ARDY of $37.3 \%$. It is demonstrated that with the same number of parameters, the solution model of this study yielded better calculation accuracy. The solution model of this study could further be simplified to a single parameter equation without significant increase in calculation error.

One advantage of the solution model approach is that even the single parameter $\alpha$ can be generalized and the solid solubility can be predicted. This study extended our previous work [9] and correlated the single parameter $\alpha$ as a function of the physical properties of the solid pharmaceutical compounds. Fig. 4 shows a plot of the $\alpha$ values from the one-parameter model in Table 3 against the logarithm of $\Delta U_{2}$ from Table 1 for each pure pharmaceutical component. A linear relationship was observed:

$\alpha=0.3579-0.3185 \ln \Delta U_{2}$

This correlation included many new solubility data of pharmaceutical compounds that were not considered in our previous work [9]. For the pharmaceutical systems in this study, the coefficient of determination (the $r^{2}$-value) for the generalization of $\alpha$ from Eq. (11) was closer to unity $\left(r^{2}=0.88\right)$ than that from

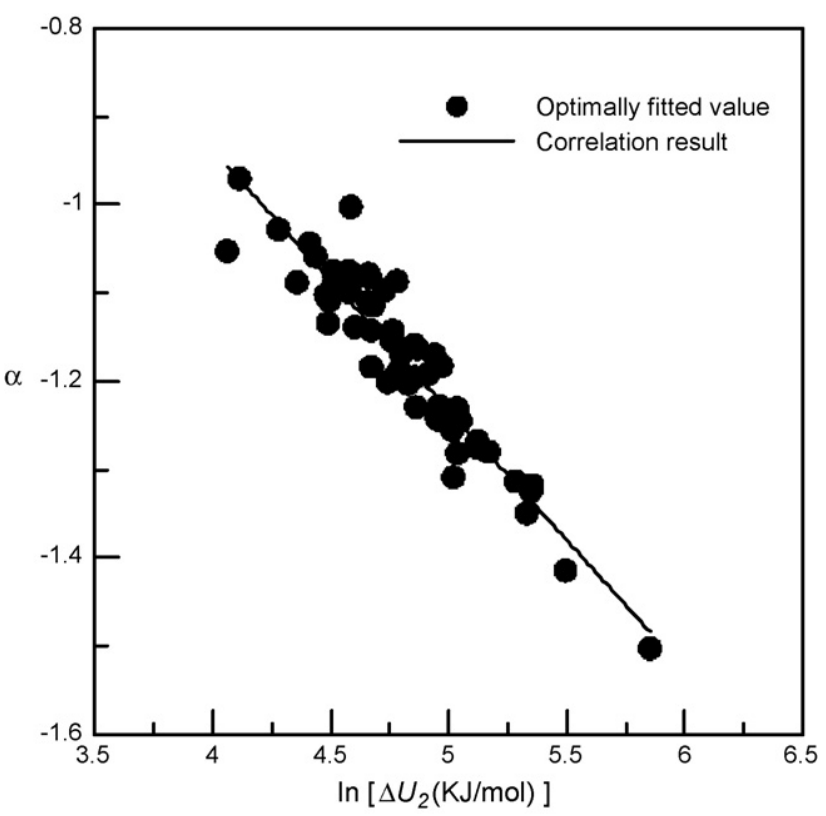

Fig. 4. Plot of the optimally fitted $\alpha$ values against the logarithm of internal energy change of vaporization for various compounds.

the previous generalization $\left(r^{2}=0.77\right)$ [9]. Fig. 5 compared the calculated solubility of Amical-48 (an organic biocide) in supercritical $\mathrm{CO}_{2}$ using Eq. (11) and the generalized equation in our previous work [9]. Since the model parameters were generalized, both calculations were predictive in nature. It was demonstrated that Eq. (11) was feasible in predicting the solid solubility, and yielded improved results to those from our previous study [9].

The prediction and comparison for solid solubility of bisacodyl (a laxative drug) in supercritical $\mathrm{CO}_{2}$ is shown in Fig. 6. The ARDY for this prediction was $25.6 \%$ and was comparable to that from the single-parameter result shown in Table 3. The generalized correlation shown in Eq. (11) has further been

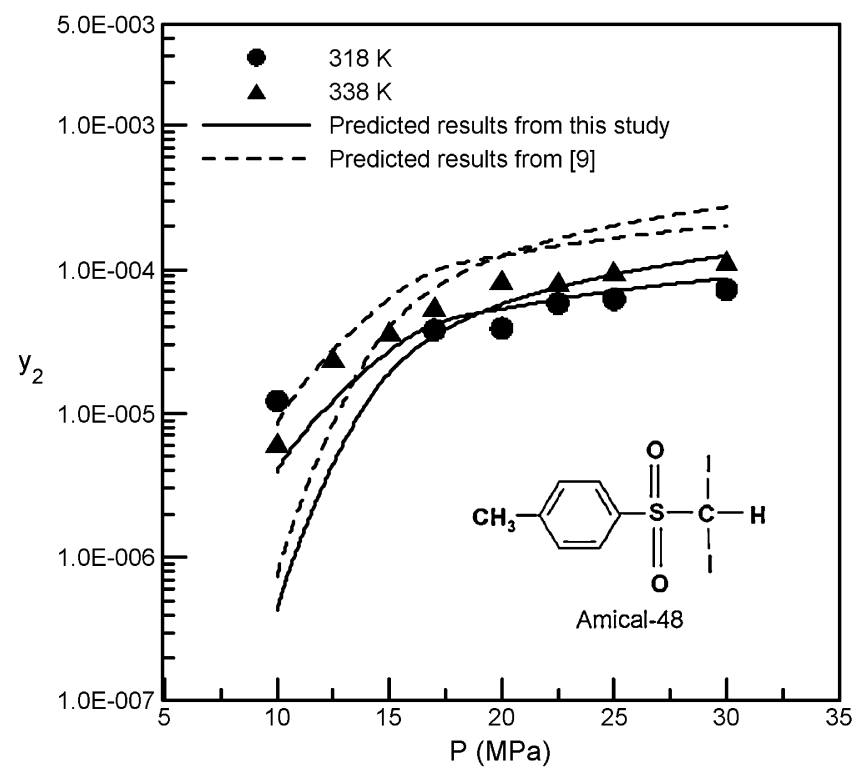

Fig. 5. Comparison for the predicted solid solubility of Amical-48 (2) in supercritical $\mathrm{CO}_{2}$ (1) using two solution models. 


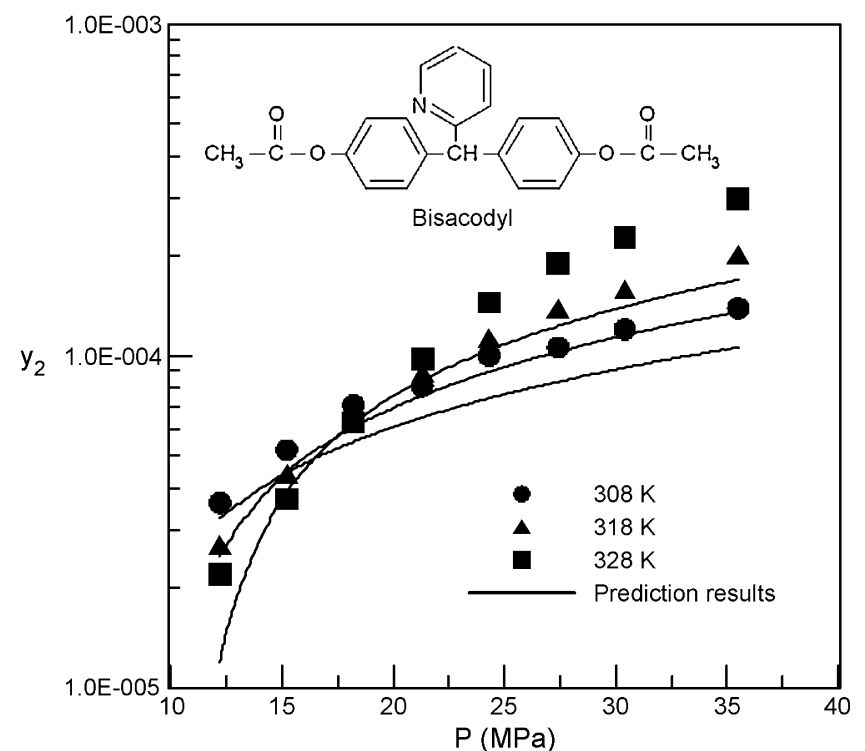

Fig. 6. Predicted solid solubility of bisacodyl (2) in supercritical $\mathrm{CO}_{2}$ (1) using the generalized correlation in this study.

employed to predict the solid solubility of pharmaceutical compounds that were not originally included in Table 1. Fig. 7 shows the prediction results of flavone at different temperatures. The ARDY for this prediction was $49.2 \%$ where only limited estimated values of pure solid properties were used in this calculation. Due to the diversification and complexity of pharmaceutical compounds, the simple generalized correlation shown in Eq. (11) yielded acceptable accuracy $(\mathrm{ARDY}=58 \%$ ) for 30 systems listed in Table 1 . The generalized equation from our previous study [9] could also predict the solid solubilities for half of the systems listed in Table 1 with an ARDY of 64\%. Eq. (11) of this study, however, gave smaller peak deviations for pharmaceutical compounds. Further classification of pharmaceutical compounds according to specific functional groups, and

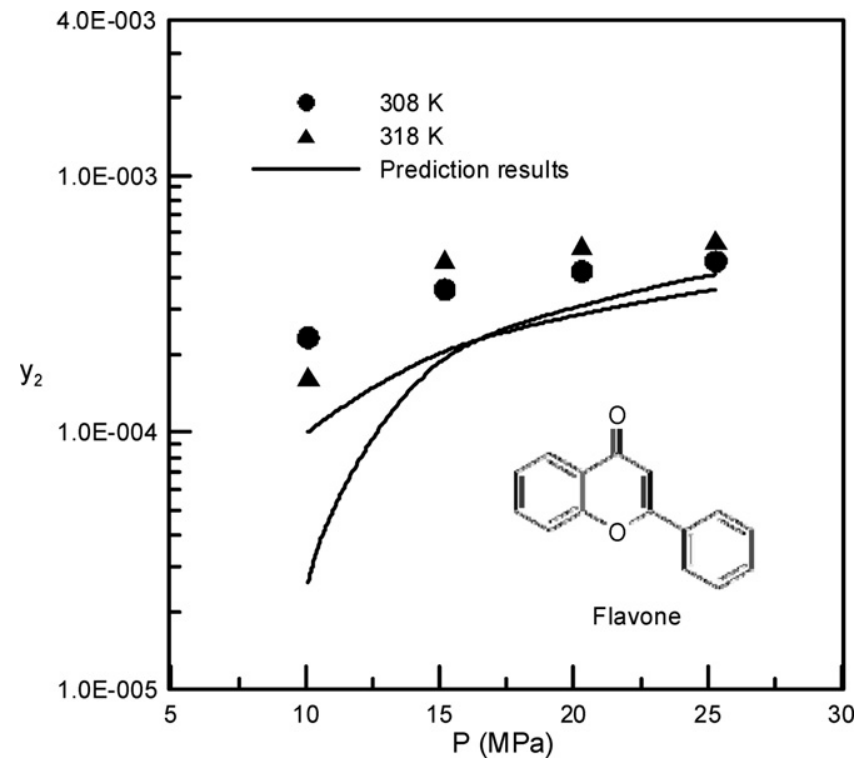

Fig. 7. Predicted solid solubility of flavone (2) in supercritical $\mathrm{CO}_{2}$ (1) using the generalized correlation in this study. generalized correlation equations for each group are required when more experimental data are reported. Applying Eq. (11), it also showed that the solid solubilities in supercritical $\mathrm{CO}_{2}$ could be predicted within the correct order of magnitude for $70 \%$ of the systems listed in Table 1. These results demonstrated the superior prediction capability of the solution model where the semi-empirical methods shown in Table 3 did not provide.

Based on our calculation results, correlation for the solubilities of pharmaceutical compounds in supercritical $\mathrm{CO}_{2}$ with only one adjustable parameter $\alpha$ is the best choice. If we have one or two experimental solid solubility data, the $\alpha$ value can be determined and further used in estimating the solubilities at other temperature or pressure conditions.

\section{Conclusion}

The solid solubilities of pharmaceutical compounds in supercritical $\mathrm{CO}_{2}$ were correlated using the solution model. The correlation results were also compared with those from three commonly used semi-empirical equations. The solution model with dual or single adjustable parameter yielded absolute relative deviation (ARDY) in solid solubility of $16.5 \%$ and $23.7 \%$, respectively. These results were better than those from the semi-empirical equations with the same number of adjustable parameters. The optimally fitted parameters of this study were further generalized and used for predicting the solubilities of pharmaceutical compounds in supercritical $\mathrm{CO}_{2}$. The prediction results showed an ARDY of 58\% for half of the systems in this study. Further improvement for the generalized correlation based on specific functional groups is required when more experimental data are reported.

\section{Acknowledgement}

The authors are grateful to the support of this study from the National Science Council, Republic of China.

\section{References}

[1] E.J. Beckman, J. Supercrit. Fluids 28 (2004) 121-191.

[2] E. Reverchon, R. Adami, J. Supercrit. Fluids 37 (2006) 1-22.

[3] J. Jung, M. Perrut, J. Supercrit. Fluids 20 (2001) 179-219.

[4] J. Chrastil, J. Phys. Chem. 86 (1982) 3016-3021.

[5] J. Mendez-Santiago, A.S. Teja, Fluid Phase Equilib. 158-160 (1999) 501-510.

[6] M. Zhong, B. Han, J. Ke, H. Yan, D.Y. Peng, Fluid Phase Equilib. 146 (1998) 93-102.

[7] Y. Iwai, Y. Koga, T. Fukuda, Y. Arai, J. Chem. Eng. Jpn. 25 (1992) 757-760.

[8] D. Bush, C.A. Eckert, Fluid Phase Equilib. 150-151 (1998) 479-492.

[9] J.S. Cheng, M. Tang, Y.P. Chen, Fluid Phase Equilib. 194-197 (2002) 483-491.

[10] S.H. Yalkowsky, Ind. Eng. Chem. Fundam. 18 (1979) 108-111.

[11] D.Y. Peng, D.B. Robinson, Ind. Eng. Chem. Fundam. 15 (1976) 59-64.

[12] J.F. Ely, W.M. Haynes, B.C. Bain, J. Chem. Thermodyn. 21 (1989) 879-894.

[13] R.F. Fedors, Polym. Eng. Sci. 14 (1974) 147-154.

[14] I. Medina, J.L. Bueno, J. Chem. Eng. Data 46 (2001) 1211-1214.

[15] K. Matsuyama, K. Mishima, R. Ohdate, M. Chidori, H. Yang, J. Chem. Eng. Data 48 (2003) 1040-1043.

[16] G. Madras, C. Erkey, A. Akgerman, J. Chem. Eng. Data 38 (1993) 422-423.

[17] K.G. Joback, R.C. Reid, Chem. Eng. Comm. 57 (1987) 233-243. 